\title{
Quasi-stationary solutions of self-gravitating scalar fields around black holes
}

\author{
Nicolas Sanchis-Gual, ${ }^{1}$ Juan Carlos Degollado, ${ }^{2}$ Pedro J. Montero, ${ }^{3}$ and José A. Font ${ }^{1,4}$ \\ ${ }^{1}$ Departamento de Astronomía y Astrofísica, Universitat de València, \\ Dr. Moliner 50, 46100, Burjassot (València), Spain \\ ${ }^{2}$ Departamento de Ciencias Computacionales, Centro Universitario \\ de Ciencias Exactas e Ingeniería, Universidad de Guadalajara \\ Av. Revolución 1500, Colonia Olímpica C.P. 44430, Guadalajara, Jalisco, Mexico \\ ${ }^{3}$ Max-Planck-Institute für Astrophysik, Karl-Schwarzschild-Str. 1, 85748, Garching bei München, Germany \\ ${ }^{4}$ Observatori Astronòmic, Universitat de València, \\ C/ Catedrático José Beltrán 2, 46980, Paterna (València), Spain
}

(Dated: December 2014)

\begin{abstract}
Recent perturbative studies have shown the existence of long-lived, quasi-stationary configurations of scalar fields around black holes. In particular, such configurations have been found to survive for cosmological timescales, which is a requirement for viable dark matter halo models in galaxies based on such type of structures. In this paper we perform a series of numerical relativity simulations of dynamical non-rotating black holes surrounded by self-gravitating scalar fields. We solve numerically the coupled system of equations formed by the Einstein and the Klein-Gordon equations under the assumption of spherical symmetry using spherical coordinates. Our results confirm the existence of oscillating, long-lived, self-gravitating scalar field configurations around non-rotating black holes in highly dynamical spacetimes with a rich scalar field environment. Our numerical simulations are long-term stable and allow for the extraction of the resonant frequencies to make a direct comparison with results obtained in the linearized regime. A byproduct of our simulations is the existence of a degeneracy in plausible long-lived solutions of Einstein equations that would induce the same motion of test particles, either with or without the existence of quasi-bound states.
\end{abstract}

PACS numbers: 95.30.Sf 04.70.Bw 04.25.dg

\section{INTRODUCTION}

Scalar fields play an important role in different areas of theoretical physics. For instance, recent models for the evolution of the universe, dark energy and string theory require the existence of ultra-light scalar degrees of freedom [1- In a cosmological context, scalar fields have been suggested as the constituents of dark matter halos in galaxies 4 4. There is large observational evidence that points to the presence of supermassive black holes in the centers of most nearby galaxies. Furthermore, the evolution of these black holes is directly connected to their host galaxy evolution 10. It has been suggested that in order to consider scalar fields as plausible candidates for dark matter halos they must survive for cosmological time scales in the presence of black holes [11].

There are several studies in the literature investigating the dynamics of scalar fields around black holes within the linearized regime, i.e. in the test field regime in which the black hole spacetime is taken as a background where the scalar field evolves [12 14. One of the key results of linearized studies concerning massive scalar fields surrounding stationary black holes is that as a result of the presence of a potential well due to the mass term, two types of modes are possible depending on the boundary conditions at infinity: no-normalizable quasi-normal modes and quasi-bound states, which decay at infinity and thus are localized around the black hole. If the black hole is spinning the latter class can yield exponentially growing modes [15, 16] and to hairy black hole solutions
17.

Quasi-bound states are scalar field configurations around black holes that may be very long lived [14. Many of their characteristics can be derived in the test field approximation. For instance, it is possible to determine their complex frequency by imposing ingoing boundary conditions at the black hole horizon and an asymptotically decay behaviour at infinity. The problem of obtaining the frequencies of the quasi-bound states reduces to an eigenvalue problem which can be solved in several efficient ways (see e.g. 18, 19]). The real part represents the oscillation frequency and the imaginary part gives their rate of decay.

While the test field approximation is valid as long as the energy content of the field is small it will break down eventually as the energy increases. At some point, the backreaction of the scalar field onto the spacetime dynamics becomes non-negligible and non-linear simulations of self-gravitating scalar field configurations are necessary. There are recent studies in this direction, most notably 20, where a fully 3D evolution of scalar fields around black holes was performed. In such work the authors made a thorough study of scalar field configurations around stationary and rotating black holes finding quasi-bound states when the amount of scalar field contributes to some fraction of the mass of the black hole. Here we focus on the cases in which the mass of the scalar field cloud may be greater than the mass of the black hole and therefore constitutes a strong deviation from the linearized regime. Stated in a different way, our setup involves a large scalar field environment in a highly 
dynamical spacetime. These scenarios may be framed as the final stage of a violent process such as the bosenova explosion 21] or as a Kerr hairy solution in which the black hole has lost its spin.

The use of spherical symmetry allows us to follow accurately the evolution of the system and describe the development of a single mode without interference effects from other modes. Furthermore, the significantly long runs we can afford $\left(t \sim 10^{5} M\right.$, where $M$ is the bare mass of the black hole) allow us to obtain very precise figures for the frequencies of the bound states. A comment on orders of magnitude becomes necessary at this point as it should be noted that the frequency of the quasi-bound states for very small values of the scalar field mass $M \mu$ compatible with dark matter models, is of the order of $M \omega \sim 10^{-6}$ in geometrized units ${ }^{1}$. According to our results, in order to capture accurately the values of the corresponding frequencies one would have to evolve the system at least for $t \sim 10^{10} M$. Such timescale, however, cannot be reached even at the linear level. For this reason we will consider values of $\mu$ in the range from 0.08 to 0.3 as we discuss below.

For this work we have coupled the Klein-Gordon equation to the Einstein equations taking advantage of the computational framework recently proposed by Montero and Cordero-Carrión [23] in which a secondorder partially-implicit Runge-Kutta (PIRK) method is applied to the Baumgarte-Shapiro-Shibata-Nakamura (BSSN) formulation of the Einstein equations. This approach has been shown to lead to long-term stable numerical simulations of both vacuum and non-vacuum (with fluids) spacetimes using spherical coordinates without the need for a regularization algorithm at the origin.

The paper is organized as follows: Section II describes the formulation of the coupled Einstein-Klein-Gordon system and the construction of the initial data. Section III focuses on the time integration of the system of evolution equations. Our results are presented and discussed in Section IV] Finally, we end with a summary of our findings in Section $\mathrm{V}$. In the following sections Greek indices $\alpha, \beta, \ldots$ run over spacetime indices, while Latin indices $i, j, \ldots$ run over space indices only. Throughout this article we use geometrized units $c=G=1$.

\section{BASIC EQUATIONS}

We investigate the dynamics of a self-gravitating scalar field configuration around a black hole by solving numerically the coupled Einstein-Klein-Gordon system

$$
R_{\alpha \beta}-\frac{1}{2} g_{\alpha \beta} R=8 \pi T_{\alpha \beta}
$$

\footnotetext{
${ }^{1}$ In physical units this corresponds to $\hbar \mu \sim 10^{-24} \mathrm{eV}$ [6] 22.
}

with matter content given by the stress energy tensor

$$
T_{\alpha \beta}=\partial_{\alpha} \Phi \partial_{\beta} \Phi-\frac{1}{2} g_{\alpha \beta}\left(\partial^{\sigma} \Phi \partial_{\sigma} \Phi+\mu^{2} \Phi^{2}\right)
$$

The conservation of this stress-energy tensor implies that the field obeys the Klein-Gordon equation

$$
\square \Phi-\mu^{2} \Phi=0
$$

where the D'Alambertian operator is defined by $\square:=$ $(1 / \sqrt{-g}) \partial_{\alpha}\left(\sqrt{-g} g^{\alpha \beta} \partial_{\beta}\right)$. We follow the convention that $\Phi$ is dimensionless and $\mu$ has dimensions of (length) ${ }^{-1}$.

\section{A. Einstein's equations in spherical symmetry}

We assume that the spacetime $\mathcal{M}$ can be foliated by a family of spatial slices $\Sigma_{t}$ that coincide with level surfaces of a coordinate time $t$. We denote the future-pointing unit normal on $\Sigma_{t}$ with $n^{\alpha}$ and write the spacetime metric $g_{\alpha \beta}$ as

$$
\begin{aligned}
d s^{2} & =g_{\alpha \beta} d x^{\alpha} d x^{\beta} \\
& =-\alpha^{2} d t^{2}+\gamma_{i j}\left(d x^{i}+\beta^{i} d t\right)\left(d x^{j}+\beta^{j} d t\right),
\end{aligned}
$$

where $\alpha$ is the lapse function, $\beta^{i}$ the shift vector, and $\gamma_{i j}$ the spatial metric induced on $\Sigma$,

$$
\gamma_{\alpha \beta}=g_{\alpha \beta}+n_{\alpha} n_{\beta}
$$

In terms of the lapse and the shift, the normal vector $n^{\alpha}$ can be expressed as

$$
n^{\alpha}=\left(1 / \alpha,-\beta^{i} / \alpha\right) \quad \text { or } \quad n_{\alpha}=(-\alpha, 0,0,0) .
$$

We adopt a conformal decomposition of the spatial metric $\gamma_{i j}$

$$
\gamma_{i j}=e^{4 \chi} \hat{\gamma}_{i j}
$$

where $\psi=e^{\chi}=(\gamma / \hat{\gamma})^{1 / 12}$ is the conformal factor, $\hat{\gamma}_{i j}$ the conformally related metric and $\hat{\gamma}$ its determinant.

Under the assumption of spherical symmetry the line element may be written as

$$
d s^{2}=e^{4 \chi}\left(a(t, r) d t^{2}+r^{2} b(t, r) d \Omega^{2}\right),
$$

with $d \Omega^{2}=\sin ^{2} \theta d \varphi^{2}+d \theta^{2}$ being the solid angle element and $a(t, r)$ and $b(t, r)$ the metric functions.

We set the value of $\hat{\gamma}$ at $t=0$ as that of the determinant of the flat metric in spherical coordinates $\stackrel{\circ}{\gamma}$. The evolution equations for the conformal factor and for the conformal metric components take the form

$$
\begin{aligned}
\partial_{t} X & =\beta^{r} \partial_{r} X-\frac{1}{3} X \hat{\nabla}_{m} \beta^{m}+\frac{1}{3} X \alpha K \\
\partial_{t} a & =\beta^{r} \partial_{r} a+2 a \partial_{r} \beta^{r}-\frac{2}{3} a \hat{\nabla}_{m} \beta^{m}-2 \alpha a A_{a} \\
\partial_{t} b & =\beta^{r} \partial_{r} b+2 b \frac{\beta^{r}}{r}-\frac{2}{3} b \hat{\nabla}_{m} \beta^{m}-2 \alpha b A_{b}
\end{aligned}
$$


where $X \equiv e^{-2 \chi}, K$ is the trace of the extrinsic curvature, $\hat{\nabla}_{m} \beta^{m}$ is the divergence of the shift vector $\beta^{i}, \hat{A}_{i j}$ is the traceless part of the conformal extrinsic curvature, and

$$
A_{a} \equiv \hat{A}_{r}^{r}, \quad A_{b} \equiv \hat{A}_{\theta}^{\theta}
$$

The evolution equation for $K$ is

$$
\begin{aligned}
\partial_{t} K & =\beta^{r} \partial_{r} K-\nabla^{2} \alpha+\alpha\left(A_{a}^{2}+2 A_{b}^{2}+\frac{1}{3} K^{2}\right) \\
& +4 \pi \alpha\left(\rho+S_{a}+2 S_{b}\right)
\end{aligned}
$$

Next, the evolution equation for the independent component of the traceless part of the conformal extrinsic curvature, $A_{a}$, is given by

$$
\begin{aligned}
\partial_{t} A_{a} & =\beta^{r} \partial_{r} A_{a}-\left(\nabla^{r} \nabla_{r} \alpha-\frac{1}{3} \nabla^{2} \alpha\right)+\alpha\left(R_{r}^{r}-\frac{1}{3} R\right) \\
& +\alpha K A_{a}-\frac{16}{3} \pi \alpha\left(S_{a}-S_{b}\right),
\end{aligned}
$$

where $R_{r}^{r}$ is the mixed radial component of the Ricci tensor and $R$ is its trace.

Finally, the evolution equation for $\hat{\Delta}^{r}$, the radial component of the additional BSSN variables [25] $\hat{\Delta}^{i}=$ $\hat{\gamma}^{m n} \hat{\Delta}_{m n}^{i}$ with $\hat{\Delta}_{b c}^{a}=\hat{\Gamma}_{b c}^{a}-\stackrel{\circ}{\Gamma}_{b c}^{a}$, is given by

$$
\begin{aligned}
\partial_{t} \hat{\Delta}^{r} & =\beta^{r} \partial_{r} \hat{\Delta}^{r}-\hat{\Delta}^{r} \partial_{r} \beta^{r}+\frac{1}{a} \partial_{r}^{2} \beta^{r}+\frac{2}{b} \partial_{r}\left(\frac{\beta^{r}}{r}\right) \\
& +\frac{\sigma}{3}\left(\frac{1}{a} \partial_{r}\left(\hat{\nabla}_{m} \beta^{m}\right)+2 \hat{\Delta}^{r} \hat{\nabla}_{m} \beta^{m}\right) \\
& -\frac{2}{a}\left(A_{a} \partial_{r} \alpha+\alpha \partial_{r} A_{a}\right) \\
& +2 \alpha\left(A_{a} \hat{\Delta}^{r}-\frac{2}{r b}\left(A_{a}-A_{b}\right)\right) \\
& +\frac{2 \alpha}{a}\left[\partial_{r} A_{a}-\frac{2}{3} \partial_{r} K+6 A_{a} \partial_{r} \chi\right. \\
& \left.+\left(A_{a}-A_{b}\right)\left(\frac{2}{r}+\frac{\partial_{r} b}{b}\right)-8 \pi j_{r}\right] .
\end{aligned}
$$

The matter source terms $\rho, S_{a}, S_{b}$ and $j_{r}$ appearing in the previous equations are components of the energymomentum tensor 2 given by

$$
\begin{aligned}
\rho & :=n^{\alpha} n^{\beta} T_{\alpha \beta}=\frac{1}{2}\left(\Pi^{2}+\frac{\Psi^{2}}{a e^{4 \chi}}\right)+\frac{1}{2} \mu^{2} \Phi^{2}, \\
j^{r} & :=-P^{r \alpha} n^{\beta} T_{\alpha \beta}=-\Pi \Psi, \\
S_{a} & :=T_{r}^{r}=\frac{1}{2}\left(\Pi^{2}+\frac{\Psi^{2}}{a e^{4 \chi}}\right)-\frac{1}{2} \mu^{2} \Phi^{2}, \\
S_{b} & :=T_{\theta}^{\theta}=\frac{1}{2}\left(\Pi^{2}-\frac{\Psi^{2}}{a e^{4 \chi}}\right)-\frac{1}{2} \mu^{2} \Phi^{2},
\end{aligned}
$$

where $\Pi$ and $\Psi$ are defined below. The Hamiltonian and momentum constrains are given by the following two equations that we compute to monitor the accuracy of the numerical evolutions:

$$
\begin{aligned}
\mathcal{H} & \equiv R-\left(A_{a}^{2}+2 A_{b}^{2}\right)+\frac{2}{3} K^{2}-16 \pi \rho=0 \\
\mathcal{M}^{r} & \equiv \partial_{r} A_{a}-\frac{2}{3} \partial_{r} K+6 A_{a} \partial_{r} \chi \\
& +\left(A_{a}-A_{b}\right)\left(\frac{2}{r}+\frac{\partial_{r} b}{b}\right)-8 \pi S_{r}=0 .
\end{aligned}
$$

\section{Gauge conditions}

In addition to the BSSN spacetime variables, there are two more variables left undetermined, the lapse function $\alpha$, and the shift vector $\beta^{i}$. Our code can handle arbitrary gauge conditions and for the simulations reported in this paper we use the so called "non-advective $1+\log$ " condition [26] for the lapse, and a variation of the "Gammadriver" condition for the shift vector [25, 27].

The form of this slicing condition is expressed as

$$
\partial_{t} \alpha=-2 \alpha K \text {. }
$$

For the radial component of the shift vector, we choose the Gamma-driver condition, which is written as

$$
\begin{aligned}
\partial_{t} B^{r} & =\frac{3}{4} \partial_{t} \hat{\Delta}^{r}, \\
\partial_{t} \beta^{r} & =B^{r},
\end{aligned}
$$

where the auxiliary variable $B^{r}$ is introduced.

\section{B. Klein-Gordon equation}

In order to solve the Klein-Gordon equation we use two first order variables defined as:

$$
\begin{aligned}
& \Pi:=n^{\alpha} \partial_{\alpha} \Phi=\frac{1}{\alpha}\left(\partial_{t} \Phi-\beta^{r} \partial_{r} \Phi\right) \\
& \Psi:=\partial_{r} \Phi .
\end{aligned}
$$

Therefore, using equation (3) we obtain the following system of first order equations:

$$
\begin{aligned}
\partial_{t} \Phi & =\beta^{r} \partial_{r} \Phi+\alpha \Pi, \\
\partial_{t} \Psi & =\beta^{r} \partial_{r} \Psi+\Psi \partial_{r} \beta^{r}+\partial_{r}(\alpha \Pi), \\
\partial_{t} \Pi & =\beta^{r} \partial_{r} \Pi+\frac{\alpha}{a e^{4 \chi}}\left[\partial_{r} \Psi\right. \\
& \left.+\Psi\left(\frac{2}{r}-\frac{\partial_{r} a}{2 a}+\frac{\partial r b}{b}+2 \partial_{r} \chi\right)\right] \\
& +\frac{\Psi}{a e^{4 \chi}} \partial_{r} \alpha+\alpha K \Pi-\alpha \mu^{2} \Phi .
\end{aligned}
$$

\section{Initial data: solving the constraints}

It has been shown in 20] that choosing the maximal slicing condition and a vanishing scalar field $\Phi$, the momentum constraint is satisfied trivially and the Hamiltonian constraint can be solved analytically. In order to 
make the process more dynamic, and allow the black hole mass to grow rapidly, we choose instead as initial data a Gaussian distribution for the scalar field of the form

$$
\Phi=A_{0} e^{\left(r-r_{0}\right)^{2} / \lambda^{2}}
$$

with $A_{0}$ the initial amplitude, $r_{0}$ the center of the Gaussian and $\lambda$ its width. The auxiliary first order quantities are initialized as follows

$$
\begin{aligned}
& \Pi(t=0, r)=0, \\
& \Psi(t=0, r)=2 \frac{\left(r-r_{0}\right)}{\lambda^{2}} A_{0} e^{\left(r-r_{0}\right)^{2} / \lambda^{2}} .
\end{aligned}
$$

We choose a conformally flat metric with $a=b=1$ together with a time symmetry condition $K_{i j}=0$. With these choices and $\Pi(t=0, r)=0$ the momentum constraint is satisfied trivially and the Hamiltonian constraint 20 gives an equation for the conformal factor $\psi=e^{\chi}$,

$$
\partial_{r r} \psi+\frac{2}{r} \partial_{r} \psi+2 \pi \psi^{5} \rho=0 .
$$

In order to solve this equation for a back hole, we assume that the conformal factor can be written in a puncturelike form as

$$
\psi=1+\frac{M}{2 r}+u(r),
$$

and we then substitute this form in the Hamiltonian constraint 20 to obtain

$$
\partial_{r r} u(r)+\frac{2}{r} \partial_{r} u(r)+2 \pi \psi^{5} \rho=0 .
$$

Given a distribution of the scalar field density $\rho$, we solve this ordinary differential equation for $u$ using a standard four order Runge-Kutta integrator, assuming that $u \rightarrow 0$ as $r \rightarrow \infty$ and regularity at the origin.

\section{TIME INTEGRATION}

We employ the second-order PIRK method developed by [28] to integrate the evolution equations in time (from time $t^{n}$ to $t^{n+1}$ ) and to handle the singular terms that appear in the evolution equations due to our choice of curvilinear coordinates. Writing a system of PDEs as follows

$$
\left\{\begin{array}{l}
u_{t}=\mathcal{L}_{1}(u, v) \\
v_{t}=\mathcal{L}_{2}(u)+\mathcal{L}_{3}(u, v),
\end{array}\right.
$$

where $\mathcal{L}_{1}, \mathcal{L}_{2}$ and $\mathcal{L}_{3}$ represent general non-linear differential operators, the second-order PIRK method takes the following form:

$$
\left\{\begin{array}{l}
u^{(1)}=u^{n}+\Delta t L_{1}\left(u^{n}, v^{n}\right), \\
v^{(1)}=v^{n}+\Delta t\left[\frac{1}{2} L_{2}\left(u^{n}\right)+\frac{1}{2} L_{2}\left(u^{(1)}\right)+L_{3}\left(u^{n}, v^{n}\right)\right],
\end{array}\right.
$$

$$
\left\{\begin{array}{rl}
u^{n+1}=\frac{1}{2}\left[u^{n}+u^{(1)}+\Delta t L_{1}\left(u^{(1)}, v^{(1)}\right)\right], \\
v^{n+1}=v^{n}+\frac{\Delta t}{2} & {\left[L_{2}\left(u^{n}\right)+L_{2}\left(u^{n+1}\right)\right.} \\
& \left.+L_{3}\left(u^{n}, v^{n}\right)+L_{3}\left(u^{(1)}, v^{(1)}\right)\right]
\end{array},\right.
$$

where we denote by $L_{1}, L_{2}$ and $L_{3}$ the corresponding discrete operators. In particular, we note that $L_{1}$ and $L_{3}$ will be treated in an explicit way, whereas the $L_{2}$ operator will contain the singular terms appearing in the sources of the equations and, therefore, will be treated partially implicitly.

This scheme is applied to the Klein-Gordon equation and to the BSSN evolution equations. We include all the problematic terms appearing in the sources of the equations, that is stiff source terms that can lead to the development of numerical instabilities (e.g., $1 / r$ factors due to spherical coordinates close to $r=0$ even when regular data is evolved), in the $L_{2}$ operator. Firstly, the conformal metric components $a$ and $b$, the quantity $X$ (function of the conformal factor), the lapse function $\alpha$, the radial component of the shift $\beta^{r}$, and the scalar field $\Phi$, are evolved explicitly, i.e. as the generic variable $u$ is evolved in the previous PIRK scheme. Secondly, the traceless part of the extrinsic curvature $A_{a}$, and the trace of the extrinsic curvature $K$, are evolved partially implicitly, using updated values of $\alpha, a$ and $b$. Then, the quantity $\hat{\Delta}^{r}$, and the auxiliary first order quantities of the scalar field, $\Psi$ and $\Pi$, are evolved partially implicitly, using the updated values of $\alpha, a, b, \beta^{r}, \psi, A_{a}, K$, and $\Phi$. Finally, $B^{r}$ is evolved partially implicitly, using the updated values of $\hat{\Delta}^{r}$. We note that the matter source terms are always included in the explicitly treated parts. In the Appendix A, we give the exact form of the source terms included in each operator.

\section{NUMERICAL RESULTS}

\section{A. Initial models and convergence}

We set up puncture-like initial data representing a stationary black hole with a surrounding scalar field cloud. Whereas part of the initial scalar field is absorbed by the black hole, another part lingers for a long time in the form of a long-lived quasi-bound state which continuously falls into the black hole. We have set up and evolved 25 different models exploring different values of the initial amplitude of the pulse $A_{0}=\{0.0005,0.005,0.05,0.1\}$ and different values of the scalar field mass $M \mu=\{0.08: 0.3\}$ to study their influence in the evolution. Regarding the initial pulse, all models are placed at the same initial location $r_{0}=10 M$ and share the same width $\lambda=5$. The values for the amplitude are chosen so that the selfgravitation of the scalar field becomes important, far away from the test field regime. The bare mass of the black hole is initially set to $M=1$.

The grid resolution is set to $\Delta r=0.2 M$ and the step 
TABLE I: Initial parameters for the scalar field around the black hole with initial bare mass $M=1$. The initial Gaussian is located at $r_{0}=10 M$ with half-width $\lambda=5$. In all cases the extraction radius is $r_{\text {ext }}=100 M$. $M_{\mathrm{AH}}$ corresponds to the final mass of the apparent horizon of the black hole, $M_{\mathrm{ADM}}$ is the initial ADM mass of the system and $E_{0}$ is the initial energy of the scalar field.

\begin{tabular}{|c|c|c|c|c|c|c|c|c|}
\hline \multirow[t]{2}{*}{ Model } & \multirow[t]{2}{*}{$M \mu$} & \multirow[t]{2}{*}{$A_{0}$} & \multicolumn{3}{|c|}{$M \omega$} & \multirow[t]{2}{*}{$M_{\mathrm{AH}}$} & \multirow[t]{2}{*}{$M_{\mathrm{ADM}}$} & \multirow[t]{2}{*}{$E_{0}$} \\
\hline & & & 1 & 2 & 3 & & & \\
\hline 1 & 0.08 & 0.1 & 0.09608 & 0.09772 & - & 2.03 & 3.23 & 2.28 \\
\hline 2 & 0.09 & 0.1 & 0.10876 & 0.11063 & - & 2.07 & 3.31 & 2.37 \\
\hline 3 & 0.10 & 0.0005 & 0.09946 & 0.09985 & - & 1.0 & 1.000062 & $6.4 \mathrm{E}-6$ \\
\hline 4 & 0.10 & 0.005 & 0.09948 & 0.09989 & - & 1.0 & 1.0062 & $6.4 \mathrm{E}-6$ \\
\hline 5 & 0.10 & 0.05 & 0.09984 & 0.10455 & 0.10535 & 1.20 & 1.614 & 0.64 \\
\hline 6 & 0.10 & 0.1 & 0.12117 & 0.12412 & - & 2.18 & 3.40 & 2.46 \\
\hline 7 & 0.11 & 0.1 & 0.13382 & - & - & 2.23 & 3.50 & 2.56 \\
\hline 8 & 0.12 & 0.1 & 0.15253 & - & - & 2.36 & 3.61 & 2.66 \\
\hline 9 & 0.13 & 0.1 & 0.16734 & - & - & 2.48 & 3.73 & 2.79 \\
\hline 10 & 0.14 & 0.1 & 0.18266 & - & - & 2.55 & 3.85 & 2.91 \\
\hline 11 & 0.15 & 0.0005 & 0.14797 & 0.14951 & 0.14981 & 1.0 & 1.00008 & $8.2 \mathrm{E}-5$ \\
\hline 12 & 0.15 & 0.005 & 0.14806 & 0.14957 & 0.14981 & 1.0 & 1.008 & $8.2 \mathrm{E}-3$ \\
\hline 13 & 0.15 & 0.05 & 0.15062 & 0.15983 & 0.16043 & 1.37 & 1.77 & 0.8 \\
\hline 14 & 0.15 & 0.1 & 0.19865 & - & - & 2.68 & 3.98 & 3.04 \\
\hline 15 & 0.16 & 0.1 & 0.21529 & - & - & 2.82 & 4.13 & 3.19 \\
\hline 16 & 0.17 & 0.1 & 0.23257 & - & - & 2.96 & 4.27 & 3.34 \\
\hline 17 & 0.18 & 0.1 & 0.25079 & - & - & 3.01 & 4.43 & 3.49 \\
\hline 18 & 0.19 & 0.1 & 0.26980 & - & - & 3.25 & 4.59 & 3.65 \\
\hline 19 & 0.20 & 0.0005 & 0.19508 & 0.19883 & 0.19949 & 1.0 & 1.0001 & $1.1 \mathrm{E}-4$ \\
\hline 20 & 0.20 & 0.005 & 0.19513 & 0.19902 & 0.19973 & 1.0 & 1.010 & $1.1 \mathrm{E}-2$ \\
\hline 21 & 0.20 & 0.05 & 0.20147 & 0.21867 & - & 1.57 & 1.99 & 1.03 \\
\hline 22 & 0.20 & 0.1 & 0.28959 & - & - & 3.46 & 4.76 & 3.82 \\
\hline 23 & 0.30 & 0.0005 & 0.29812 & - & - & 1.0 & 1.00017 & $1.8 \mathrm{E}-4$ \\
\hline 24 & 0.30 & 0.005 & 0.29843 & - & - & 1.0 & 1.017 & $1.8 \mathrm{E}-2$ \\
\hline 25 & 0.30 & 0.05 & 0.34413 & - & - & 2.14 & 2.61 & 1.67 \\
\hline
\end{tabular}

time is given by $\Delta t=0.5 \Delta r$ or $\Delta t=0.3 \Delta r$. The second choice is needed to obtain long-term stable simulations for the models with larger amplitude. The final time of the numerical evolutions varies between $2 \times 10^{4} M$ for the large amplitude models and about $6 \times 10^{4} M$ for the small amplitude ones. The outer boundary of the computational domain is placed at $r=6 \times 10^{4} M$, far enough so it does not affect the dynamics in the inner region.

In Table I we present the different choices for the initial parameters of the models we have evolved, that is, the mass of the scalar field particle $M \mu$ and the amplitude of the pulse $A_{0}$. The frequencies $M \omega$ of the dominant modes are labeled according to their strength on the power spectrum. The table also contains the mass of the apparent horizon $(\mathrm{AH})$ at the end of the simulation $M_{\mathrm{AH}}$, defined as $M_{\mathrm{AH}}=\sqrt{\mathcal{A} / 16 \pi}$, where $\mathcal{A}$ is the area of the $\mathrm{AH}$, the Arnowitt-Deser-Misner (ADM) mass $M_{\mathrm{ADM}}$ of the whole system

$$
M_{\mathrm{ADM}}=-\frac{1}{2 \pi} \lim _{r \rightarrow \infty} \oint_{S} \partial_{j} \psi d S^{j}
$$

and the initial energy of the scalar field $E_{0}$

$$
E=\int_{2 M}^{\infty} \rho d V
$$

where $\rho$ is defined in Eq. 16. We have checked that the total ADM mass of the system $M_{\mathrm{ADM}}$ computed at the initial time slice using Eq. (39) is equal to the sum of the initial black hole mass plus the initial energy of the scalar field Eq. 40. The discrepancy is at most $2 \%$ of the ADM mass.

In order to test the convergence of the code we performed three simulations with different resolutions $\Delta r=$ $\{0.2 M, 0.1 M, 0.05 M\}$. In Fig. 1 we plot the rescaled evolution of the L2 norm of the Hamiltonian constraint for the particular choice of the initial amplitude $A_{0}=0.1$ and the scalar field mass $M \mu=0.1$, obtaining the expected second-order convergence of our PIRK timeevolution scheme. The same result is achieved irrespective of the model. 


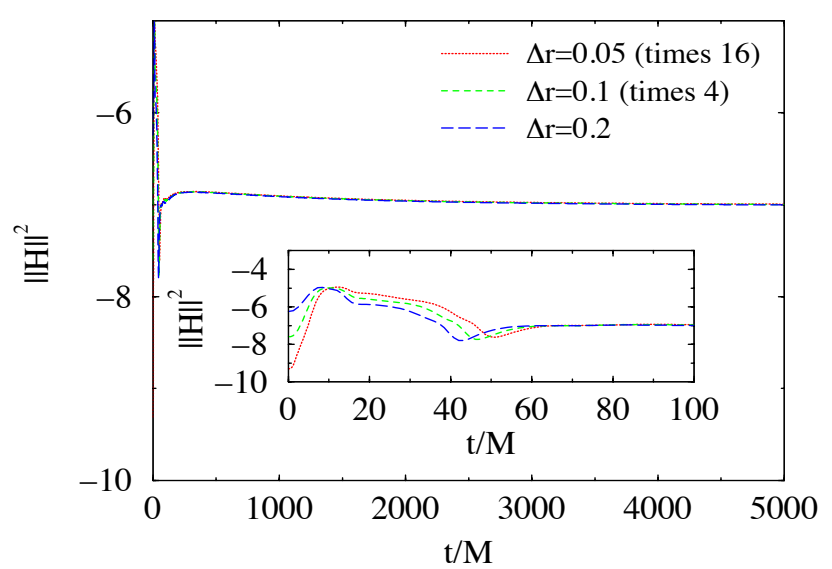

FIG. 1: Time evolution of the L2 norm of the Hamiltonian constraint for an initial scalar field pulse with $A_{0}=0.1$ and mass $M \mu=0.1$. The plot shows results for three different resolutions, rescaled by the factors corresponding to secondorder convergence. The inset shows a magnified view of the initial $100 \mathrm{M}$ in the evolution. At $t=0$ the convergence is to fourth order.

\section{B. Non-linear quasi-bound states}

We evolve the Einstein-Klein-Gordon system using the initial data given by Eqs. (30)-(32). In order to analyze the results of the simulations we extract a time series for the scalar field amplitude at an observation point with a fixed radius $r_{\text {ext }}$. Thus, to identify the frequencies at which the field oscillates we perform a Fast Fourier transform after a given number of time steps and obtain the power spectrum. For some models we were able to obtain not only the fundamental frequency of oscillation but also some of the overtones.

It is well known that the frequency resolution is inversely proportional to the simulation time and hence longer runs yield to more accurate frequencies. Thanks to the computational advantage provided by spherical symmetry and to the use of the PIRK method we were able to compute the frequencies at which the scalar field oscillates, confirming the existence of long-lived spherical states in our nonlinear evolutions even for large initial amplitudes of the scalar field.

The power spectrum obtained from the Fourier transform shows a set of distinct frequencies. In order to validate our results we contrast the numerical values of the frequency of the small amplitude configurations with the ones obtained using Leaver's continued fraction technique [29] in the frequency domain in the test field approximation.

In figures 2 to 5 we plot the evolution of the scalar field seen by an observer at $r_{\text {ext }}=100 M$ for the models 3-6 of our sample along with their corresponding Fourier
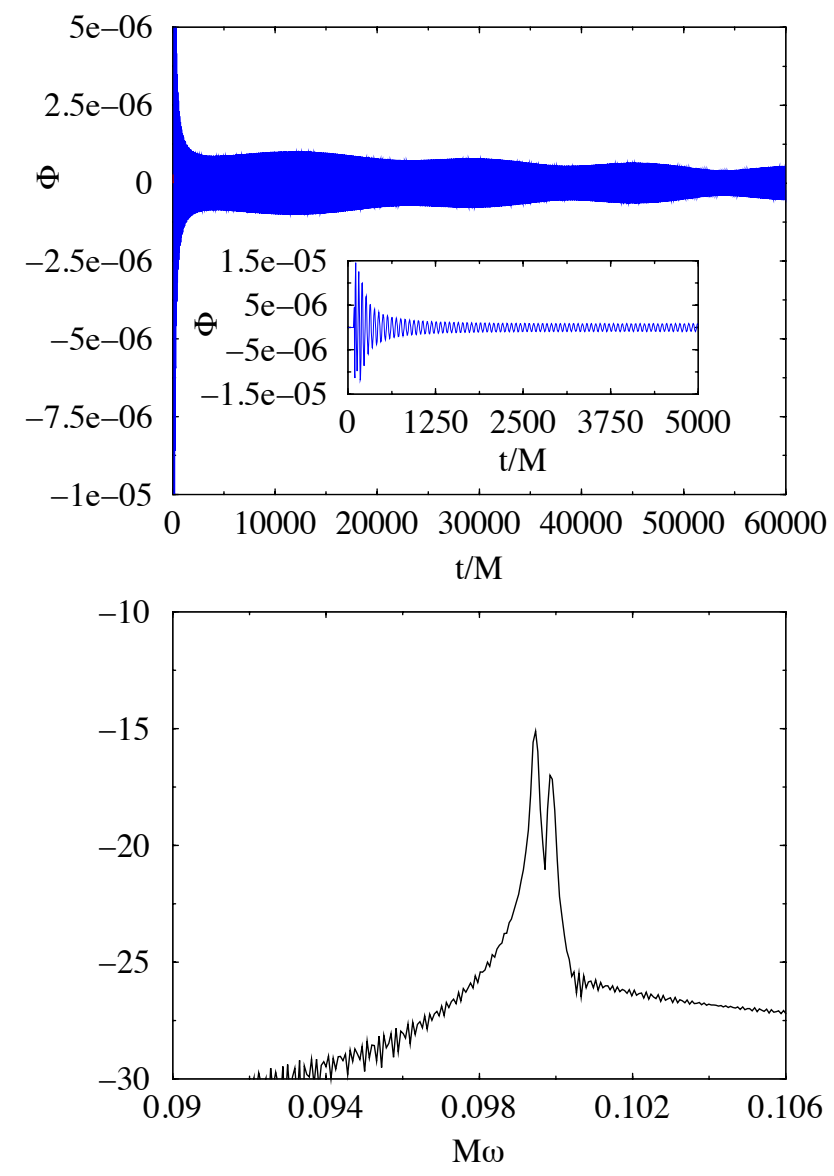

FIG. 2: Upper panel: Time evolution of the scalar field with mass $M \mu=0.1$ and $A_{0}=0.0005$ corresponding to the model 3 for an observer at $r_{\text {ext }}=100 M$. The inset shows a magnified view of the initial $5000 \mathrm{M}$ in the evolution. Lower panel: Fourier transform of the evolution of the scalar field. The units in the vertical axis are arbitrary.

transforms. In all of the plots the field is seen to be clearly oscillating. Moreover, all of these models show a distinctive beating pattern due to the presence of overtones, as described in [12, 30 32] for the case of fixed background computations and in 20] in the non-linear regime. Within the computational times we can afford, such beatings and overtones cannot be resolved only in models 7-10, 14-18, and 22-25. For the small amplitude models $3-12,14,19,20$ and 23 the frequencies are very close to the values obtained in the test field regime. As shown in Table [ the frequency grows as the amplitude is increased.

By using a matching technique, Furuhashi and Nambu showed analytically in 33 that in the limit $M \mu \ll 1$ the real part of the frequency of quasi-bound states depends 

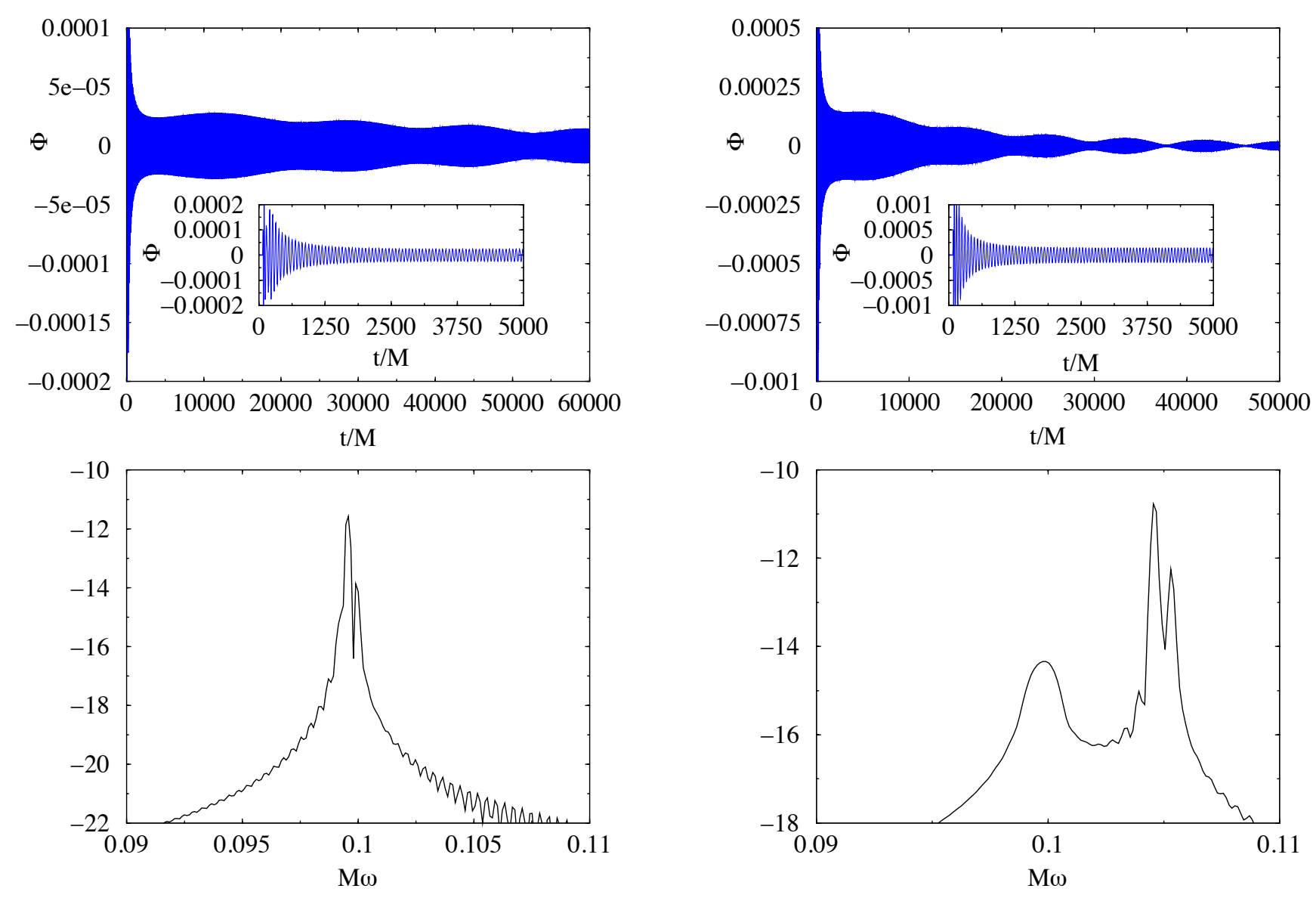

FIG. 3: Same as Fig. 2 but for model 4.

on the mass parameter $\mu$ as

$$
\omega \approx \mu\left[1-\frac{1}{2}(M \mu)^{2}\right] .
$$

In Fig. 6 we show a plot of the frequencies of all the configurations of Table 1 as a function of $\mu$. We also consider in this plot the adiabatic approximation for the frequency given by Eq. (41) for different values of the mass of the black hole $M$. For small values of $A_{0}$, the corresponding curve is indistinguishable from the one of the test field approximation. However, for greater values, the results from the non-linear approach not only deviate from the quasi-adiabatic approximation but they also follow the opposite trend. The frequency is greater than the frequency of the test field limit, whereas the adiabatic approach holds that the frequency should be smaller. This discrepancy may be due to the violation of the condition $M \mu \ll 1$ used in the analytical approximation. Nevertheless, the extrapolation of our results for the scalar field mass $\mu$ in the regime compatible with scalar field dark matter models, $M \mu \sim 10^{-6}$, supports

the validity of the model.

\section{Spacetime characterization}

Spacetime invariants are useful quantities to compare numerical solutions with known exact solutions, see for instance Ref. 34] for an instructive way to use them during the formation of a single black hole. With this aim we have used the invariants $I$ and $J$ defined as [35]

$$
\begin{aligned}
I= & \frac{1}{2}\left[\left(E_{i j} E^{i j}-B_{i j} B^{i j}\right)-2 i E_{i j} B^{i j}\right], \\
J= & -\frac{1}{6}\left[\left(E^{i}{ }_{k} E^{j k}-3 B^{i}{ }_{k} B^{j k}\right) E_{i j}\right. \\
& \left.+i\left(B_{k}^{i} B^{j k}-3 E_{k}^{i} E^{j k}\right) B_{i j}\right],
\end{aligned}
$$

to characterize our numerical spacetime. In these expressions $E_{i j}$ and $B_{i j}$ are the electric and magnetic parts of the Weyl tensor. We have computed these two quantities to use them as a measure of the deviation of our numerical solution from that corresponding to a Schwarzschild 

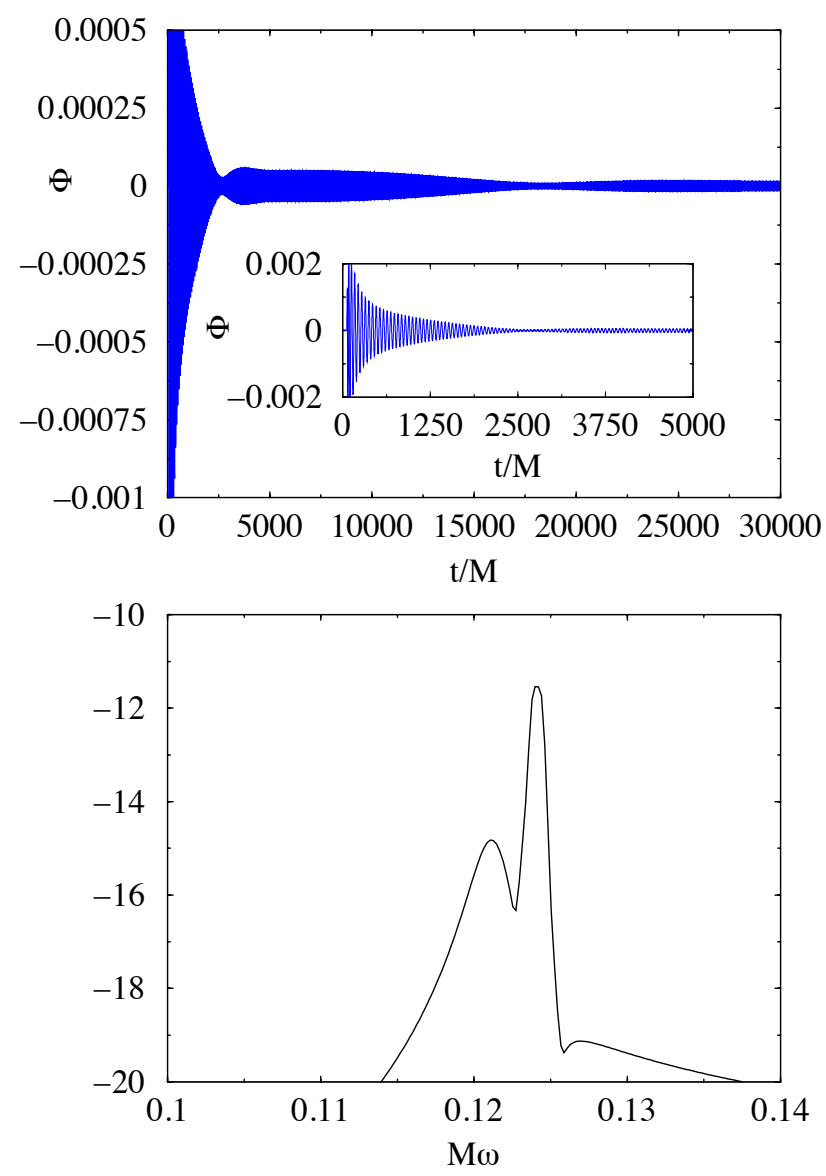

FIG. 5: Same as Fig. 2 but for model 6.

black hole. As expected, the numerical spacetime for the cases with a scalar field pulse of sufficiently small amplitude resembles the Schwarzschild solution once most of the scalar field has accreted on the black hole. The situation changes in a significant way when the amplitude increases. In the two plots of Fig. 7 the red solid curves correspond to the invariants $I$ (left panel) and $J$ (right panel) for our model 6 at $t=9000 M$. These radial profiles are compared with the corresponding purely Schwarzschild invariants for a black hole with the same final mass $\left(M_{\mathrm{AH}}=2.18\right)$, as indicated by the green dashed curves in the same figure. The deviation of our numerical spacetime from an analytic Schwarzschild black hole spacetime with equivalent mass is evident. An important remark is that both invariants may however be superimposed, at sufficiently large distances, if we choose a larger Schwarzschild black hole mass. The blue dashed curve in Fig. 7 shows that that is indeed the case if the mass of the Schwarzschild black hole is chosen to be $M_{\mathrm{AH}}=4.25$. The discrepancy is still noticeable near the black hole horizon, as the inset in Fig. 7 clearly makes visible.

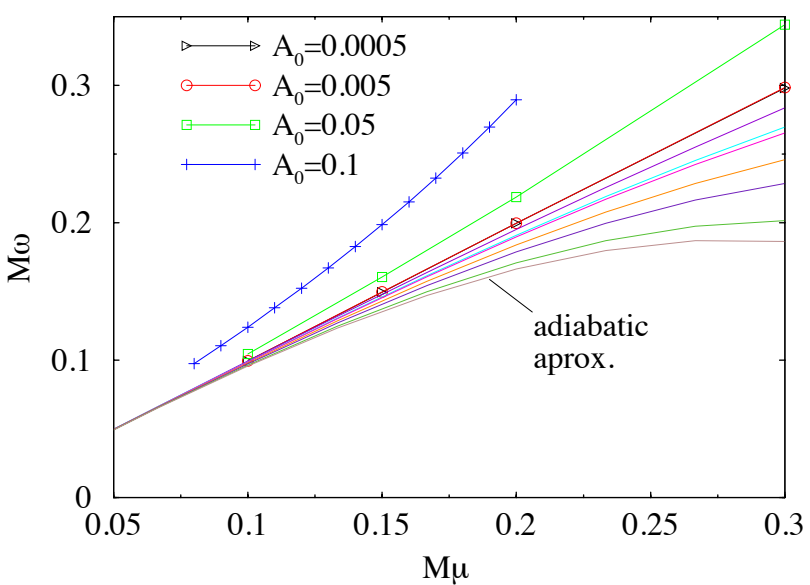

FIG. 6: Frequency $\omega$ as a function of the scalar field mass $\mu$ for the models shown in Table I] The semi-analytic relation (41) for several values of $M$ is also plotted. For models with small initial $A_{0}$ the numerical frequencies match the values of the semi analytical values showing consistency with the test field approximation and the limit $M \mu \ll 1$.

TABLE II: Mass of the black hole spacetimes corresponding to the six largest amplitude models of our sample. Columns three and four indicate, respectively, the masses of our numerically evolved spacetimes and those of the analytic Schwarzschild black holes that would induce the same radial profiles (degeneracy) in the Weyl invariants.

\begin{tabular}{ccccc}
\hline \hline Model & $M \mu$ & $M_{\mathrm{AH} 1}$ & $M_{\mathrm{AH} 2}$ & $M_{\mathrm{AH} 1} / M_{\mathrm{AH} 2}$ \\
\hline 6 & 0.10 & 2.18 & 4.25 & 1.95 \\
8 & 0.12 & 2.36 & 5.0 & 2.12 \\
10 & 0.14 & 2.55 & 6 & 2.35 \\
15 & 0.16 & 2.82 & 7.25 & 2.57 \\
17 & 0.18 & 3.1 & 8.75 & 2.82 \\
22 & 0.20 & 3.46 & 11.0 & 3.18 \\
\hline \hline
\end{tabular}

This difference may have important implications when studying the motion of test particles around black holes. In particular, there would be a degeneracy in the characterization of the underlying spacetime generating the gravitational attraction of a test particle. One observer at infinity might infer, due to the movement of a test particle, that the central object is either one isolated Schwarzschild black hole with mass $M_{1}$ or a transient (non-static) state made up of a scalar cloud around a black hole with mass $M_{2}$. These two possibilities can be regarded as well as two different, very long-lived solutions of Einstein equations that induce the same motion of test particles, either the vacuum Schwarzschild solution or that of a dynamical spherically-symmetric black hole surrounded by a scalar field.

In Table $\amalg$ we summarize the results for all the 6 cases of our sample of models with the largest initial ampli- 

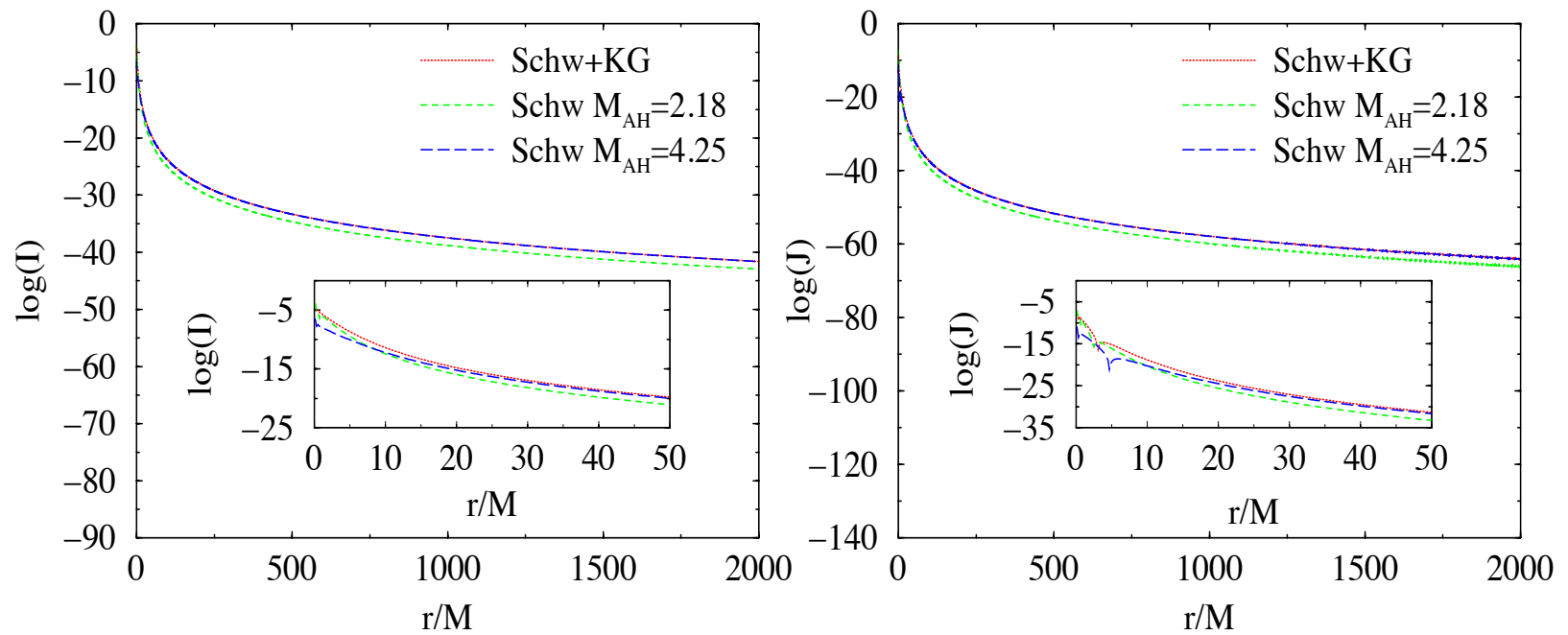

FIG. 7: Radial profiles of the Weyl invariants $I$ (left) and $J$ (right) for model 6 in Table $\mathbb{I}$ at $t=9000 M$. The red curve corresponds to our numerically evolved spacetime while the green and blue curves correspond to analytic (static) Schwarzschild spacetimes of mass $M_{\mathrm{AH}}=2.18$ and 4.25 , respectively. The inset shows a region close to the black hole horizon where the numerical solution differs considerably from a stationary Schwarzschild black hole.

tudes and for which the aforementioned degeneracy is most apparent. The third column corresponds to the final numerical black hole mass, $M_{\mathrm{AH} 1}$. The fourth column reports the equivalent Schwarzschild mass $M_{\mathrm{AH} 2}$ that would induce the same radial profiles of the Weyl invariants at large distances. The second mass is extracted by fitting the invariants at large distances and therefore it has not to be taken as an accurate measure but rather as an approximate value. The ratio $M_{\mathrm{AH} 1} / M_{\mathrm{AH} 2}$ grows with $M \mu$, as shown in the fifth column of the table. We note that it remains to be seen whether the spacetime degeneracy found in our simulations is still important, or even exists in the first place, for values of the scalar field mass $\mu$ compatibles with dark matter models, namely $M \mu \sim 10^{-6}$. As mentioned in the Introduction the timescales needed to computationally disclose the answer cannot be reached even with linear perturbation codes.

\section{CONCLUSIONS}

In this paper we have solved numerically the EinsteinKlein-Gordon system in spherical symmetry using spherical coordinates with a PIRK numerical scheme. Our study has been focused on investigating within a fully non-linear setup whether quasi-stationary scalar field configurations may be found in the form of clouds (or hairy "wigs") surrounding dynamical black holes. Such configurations have been recently put forward by 13,14 as a plausible model to describe dark matter halos in galaxies. Through a computational study based on perturbative approaches, these authors found indeed such configurations to be long-lived.

In our non-linear study we have performed a large number of (second-order) accurate and long-term stable simulations of dynamical non-rotating black holes surrounded by self-gravitating scalar fields. The models of our sample have been suitably parameterized to span a broad range of cases from the test field regime to the fully non-linear regime. Confirming earlier findings from perturbation theory we have found that also in the case of highly dynamical spacetimes (i.e. those consisting of a black hole and a rich scalar field environment) there are states which closely resemble the quasi-bound states in the test field approximation of [13, 14. In addition, we have been able to characterize the resulting spacetimes by analyzing the Weyl invariants $I$ and $J$ and have compared them to analytical values of Schwarzschild black hole spacetimes. Our results have revealed a degeneracy in plausible long-lived solutions of Einstein equations that induce the same motion of test particles, either with or without the existence of quasi-bound states. By performing a Fourier transform of the time series of our numerical data we have been able to characterize the scalar field states by their distinctive oscillation frequencies. It has been found that the scalar field oscillates with different well-defined frequencies, and that its time evolution produces in most of our models a characteristic beating pattern due to the non-linear combination of two frequencies with close enough values. Such scalar 
field oscillations may have important imprints in a number of astrophysical scenarios. For instance, the fully three-dimensional dynamical evolutions performed in 20$]$ showed that the interaction of the scalar field with the central black hole results in both scalar field and gravitational radiation. Recently [36], using first-order perturbation theory, found that the gravitational response is already present even at the linear level. Since our current study is limited to spherical symmetry, we could not perform such analysis in the present work, a task which we defer to a future investigation.

\section{Acknowledgements}

This work has been supported by CONACyT-México, by the Deutsche Forschungsgemeinschaft (DFG) through its Transregional Center SFB/TR7 "Gravitational Wave Astronomy", by the Spanish MINECO (AYA2013-40979$\mathrm{P}$ ) and by the Generalitat Valenciana (PROMETEOII2014-069). The computations have been performed at the Servei d'Informàtica de la Universitat de València.

\section{Appendix A: Source terms}

The evolution Eqs. (9)-(11), (13)-(15), 22)-(24) are evolved using a second-order PIRK method, described in Sec. III. In this Appendix the source terms included in the explicit or partially implicit operators are detailed.

Firstly, $a, b, X, \alpha, \beta^{r}$ and $\Phi$, are evolved explicitly, i.e., all the source terms of the evolution equations of these variables are included in the $L_{1}$ operator of the secondorder PIRK method.

Secondly, $A_{a}$ and $K$, are evolved partially implicitly, using updated values of $\alpha, a$ and $b$. More precisely, the corresponding $L_{2}$ and $L_{3}$ operators associated with the evolution equations for $A_{a}$ and $K$ read:

$$
\begin{aligned}
L_{2\left(A_{a}\right)} & =-\left(\nabla^{r} \nabla_{r} \alpha-\frac{1}{3} \nabla^{2} \alpha\right)+\alpha\left(R_{r}^{r}-\frac{1}{3} R\right) \\
L_{3\left(A_{a}\right)} & =\beta^{r} \partial_{r} A_{a}+\alpha K A_{a}-16 \pi \alpha\left(S_{a}-S_{b}\right), \\
L_{2(K)} & =-\nabla^{2} \alpha \\
L_{3(K)} & =\beta^{r} \partial_{r} K+\alpha\left(A_{a}^{2}+2 A_{b}^{2}+\frac{1}{3} K^{2}\right) \\
& +4 \pi \alpha\left(\rho+S_{a}+2 S_{b}\right) .
\end{aligned}
$$

Next, $\hat{\Delta}^{r}, \Psi$ and $\Pi$ are evolved partially implicitly, using the updated values of $\alpha, a, b, \beta^{r}, \psi, A_{a}, K$ and $\Phi$. Specifically, the corresponding $L_{2}$ and $L_{3}$ operators associated with the evolution equation for $\hat{\Delta}^{r}, \Psi$ and $\Pi$ are given by:

$$
\begin{aligned}
L_{2\left(\hat{\Delta}^{r}\right)} & =\frac{1}{a} \partial_{r}^{2} \beta^{r}+\frac{2}{b} \partial_{r}\left(\frac{\beta^{r}}{r}\right)+\frac{\sigma}{3 a} \partial_{r}\left(\hat{\nabla}_{m} \beta^{m}\right) \\
& -\frac{2}{a}\left(A_{a} \partial_{r} \alpha+\alpha \partial_{r} A_{a}\right)-\frac{4 \alpha}{r b}\left(A_{a}-A_{b}\right) \\
& +\frac{\xi \alpha}{a}\left[\partial_{r} A_{a}-\frac{2}{3} \partial_{r} K+6 A_{a} \partial_{r} \chi\right. \\
& \left.+\left(A_{a}-A_{b}\right)\left(\frac{2}{r}+\frac{\partial_{r} b}{b}\right)\right], \\
L_{3\left(\hat{\Delta}^{r}\right)} & \beta^{r} \partial_{r} \hat{\Delta}^{r}-\hat{\Delta}^{r} \partial_{r} \beta^{r}+\frac{2 \sigma}{3} \hat{\Delta}^{r} \hat{\nabla}_{m} \beta^{m} \\
& +2 \alpha A_{a} \hat{\Delta}^{r}-8 \pi j_{r} \frac{\xi \alpha}{a}, \\
L_{2(\Psi)} & =\partial_{r}(\alpha \Pi), \\
L_{3(\Psi)} & =\beta^{r} \partial_{r} \Psi+\Psi \partial_{r} \beta^{r}, \\
L_{2(\Pi)} & =\frac{\alpha}{a e^{4 \chi}}\left[\partial_{r} \Psi+\Psi\left(\frac{2}{r}-\frac{\partial_{r} a}{2 a}+\frac{\partial r b}{b}+2 \partial_{r} \chi\right)\right. \\
& +\frac{\Psi}{a e^{4 \chi}} \partial_{r} \alpha-\alpha \mu^{2} \Phi, \\
L_{3(\Pi)} & =\beta^{r} \partial_{r} \Pi+\alpha K \Pi
\end{aligned}
$$

Finally, $B^{r}$ is evolved partially implicitly, using the updated values of $\hat{\Delta}^{r}$, i.e., $L_{2\left(B^{r}\right)}=\frac{3}{4} \partial_{t} \hat{\Delta}^{r}$ and $L_{3\left(B^{r}\right)}=0$.
[1] A. Arvanitaki, S. Dimopoulos, S. Dubovsky, N. Kaloper, and J. March-Russell, Phys.Rev. D81, 123530 (2010), 0905.4720 .

[2] A. Arvanitaki and S. Dubovsky, Phys.Rev. D83, 044026 (2011), 1004.3558.

[3] M. Kamionkowski, J. Pradler, and D. G. E. Walker (2014), 1409.0549.
[4] J.-c. Hwang, Phys. Lett. B401, 241 (1997), astro$\mathrm{ph} / 9610042$.

[5] T. Matos, F. S. Guzman, and L. A. Urena-Lopez, Class. Quant. Grav. 17, 1707 (2000), astro-ph/9908152.

[6] T. Matos and L. A. Urena-Lopez, Phys.Rev. D63, 063506 (2001), astro-ph/0006024.

[7] T. Matos and L. A. Urena-Lopez, Class. Quant. Grav. 
17, L75 (2000), astro-ph/0004332.

[8] D. J. E. Marsh and P. G. Ferreira, Phys. Rev. D82, 103528 (2010), 1009.3501.

[9] W. Hu, R. Barkana, and A. Gruzinov, Phys. Rev. Lett. 85, 1158 (2000), astro-ph/0003365.

[10] J. Kormendy and K. Gebhardt, AIP Conference Proceedings: 20th Texas Symposium 586, 363 (2001).

[11] J. Barranco, A. Bernal, J. C. Degollado, A. Diez-Tejedor, M. Megevand, et al., Phys.Rev. D84, 083008 (2011), 1108.0931.

[12] H. Witek, V. Cardoso, A. Ishibashi, and U. Sperhake, Phys.Rev. D87, 043513 (2013), 1212.0551.

[13] J. Barranco, A. Bernal, J. C. Degollado, A. Diez-Tejedor, M. Megevand, et al., Phys.Rev.Lett. 109, 081102 (2012), 1207.2153.

[14] J. Barranco, A. Bernal, J. C. Degollado, A. Diez-Tejedor, M. Megevand, et al., Phys.Rev. D89, 083006 (2014), 1312.5808

[15] S. L. Detweiler, Phys.Rev. D22, 2323 (1980).

[16] S. R. Dolan, Phys.Rev. D76, 084001 (2007), 0705.2880.

[17] C. A. R. Herdeiro and E. Radu, Phys.Rev.Lett. 112, 221101 (2014), 1403.2757.

[18] E. Berti, V. Cardoso, and A. O. Starinets, Class.Quant.Grav. 26, 163001 (2009), 0905.2975.

[19] R. Konoplya and A. Zhidenko, Rev.Mod.Phys. 83, 793 (2011), 1102.4014.

[20] H. Okawa, H. Witek, and V. Cardoso, Phys.Rev. D89, 104032 (2014), 1401.1548.

[21] H. Yoshino and H. Kodama, Prog.Theor.Phys. 128, 153 (2012), 1203.5070.

[22] A. P. Lundgren, M. Bondarescu, R. Bondarescu, and J. Balakrishna, Astrophys. J. 715, L35 (2010),
1001.0051.

[23] P. J. Montero and I. Cordero-Carrion, Phys.Rev. D85, 124037 (2012), 1204.5377.

[24] J. D. Brown, Phys. Rev. D 79, 104029 (2009), URL http: //link.aps.org/doi/10.1103/PhysRevD.79.104029.

[25] M. Alcubierre and M. D. Mendez, Gen.Rel.Grav. 43, 2769 (2011), 1010.4013.

[26] C. Bona, J. Massó, E. Seidel, and J. Stela, Phys. Rev. D 56, 3405 (1997), URL http://link. aps .org/doi/10. 1103/PhysRevD.56.3405

[27] M. Alcubierre, B. Brügmann, P. Diener, M. Koppitz, D. Pollney, E. Seidel, and R. Takahashi, Phys. Rev. D 67, 084023 (2003), URL http://link.aps .org/doi/10. 1103/PhysRevD.67.084023

[28] I. Cordero-Carrión and P. Cerdá-Durán, ArXiv e-prints (2012), 1211.5930.

[29] E. Leaver, Proc.Roy.Soc.Lond. A402, 285 (1985).

[30] S. R. Dolan (2012), 1212.1477.

[31] Z. Zhang, E. Berti, and V. Cardoso, Phys.Rev. D88, 044018 (2013), 1305.4306.

[32] J. C. Degollado and C. A. R. Herdeiro, Phys.Rev. D89, 063005 (2014), 1312.4579.

[33] H. Furuhashi and Y. Nambu, Prog.Theor.Phys. 112, 983 (2004), gr-qc/0402037.

[34] J. G. Baker and M. Campanelli, Phys.Rev. D62, 127501 (2000), gr-qc/0003031.

[35] M. Alcubierre, Introduction to $3+1$ Numerical Relativity (Oxford Univ. Press, New York, 2008), ISBN 978-0-19920567-7.

[36] J. C. Degollado and C. A. R. Herdeiro, Phys.Rev. D90, 065019 (2014), 1408.2589. 